05.3;08.2

\title{
Механизм роста монослоя на верхней грани Ga-каталитических нитевидных нанокристаллов GaAs и GaP
}

\author{
(C) А.А. Корякин ${ }^{1}$, Ю.А. Еремеев ${ }^{2}$, С.В. Федина ${ }^{3}$, В.В. Федоров ${ }^{3}$ \\ ${ }^{1}$ Санкт-Петербургский государственный университет, Санкт-Петербург, Россия \\ ${ }^{2}$ Институт проблем машиноведения РАН, Санкт-Петербург, Россия \\ ${ }^{3}$ Санкт-Петербургский национальный исследовательский \\ Академический университет им. Ж.И. Алфёрова РАН, Санкт-Петербург, Россия \\ E-mail: koryakinaa@spbau.ru
}

Поступило в Редакцию 2 сентября 2021 г.

В окончательной редакции 10 ноября 2021 г.

Принято к публикации 10 ноября 2021 г.

Исследован механизм роста монослоя на верхней грани Ga-каталитических нитевидных нанокристаллов $\mathrm{GaAs}$ и $\mathrm{GaP}$. В рамках теоретической модели определены максимальная степень заполнения монослоя за счет вещества в капле катализатора, скорость роста нитевидных нанокристаллов и содержание атомов $\mathrm{V}$ группы в капле в зависимости от условий роста. Получены оценки коэффициента переиспарения фосфора от соседних нитевидных нанокристаллов и подложки на основе сравнения теоретической и экспериментальной скорости роста Ga-каталитических нитевидных нанокристаллов GaP.

Ключевые слова: нитевидные нанокристаллы III-V, механизм роста пар-жидкость-кристалл, нуклеация.

DOI: 10.21883/PJTF.2022.04.52079.19011

В последнее время благодаря развитию методов in situ ПЭМ-исследований (ПЭМ - просвечивающая электронная микроскопия) роста нитевидных нанокристаллов (НHК) III-V по механизму пар-жидкость-кристалл (ПЖК) стало возможным изучение процесса роста верхней грани ННК, находящейся под частицей катализатора, в реальном масштабе времени [1-5]. Это стимулировало появление ряда теоретических работ, посвященных исследованию морфологии границы раздела катализатор-ННК [1-4] и механизмов роста монослоя на верхней грани ННК [5-9]. Было обнаружено, что полный цикл формирования монослоя может содержать инкубационный период либо представляет собой непрерывный процесс [6]. В первом случае скорость роста ННК ограничена нуклеацией двумерных островков на границе раздела катализатор-ННК. Во втором случае имеет место безбарьерный механизм формирования ступени. Также обнаружено, что на механизм формирования монослоя влияет морфология границы раздела катализатор-ННК. Целью настоящей работы является исследование режимов роста монослоя при формировании Ga-каталитических (автокаталитический ПЖК-рост) ННК GaAs и $\mathrm{GaP}$ методом молекулярнопучковой эпитаксии (МПЭ). Согласно работам $[6,8,10]$, в этом случае скорость роста верхней грани ННК ограничена нуклеацией и из-за низкой растворимости мышьяка и фосфора в галлии могут реализоваться следующие два режима роста монослоя. Если после нуклеации нового монослоя разница между максимальным числом атомов $\mathrm{V}$ группы в капле $N_{\mathrm{V}}$ и равновесным числом атомов $\mathrm{V}$ группы $N_{\mathrm{V} e q}$ больше, чем количество атомов $\mathrm{V}$ группы в монослое $N_{\mathrm{ML}}$, то монослой растет в „быстром режиме“, ограниченном диффузией частиц $\mathrm{V}$ группы в капле. Если выполнено обратное неравенство, т. е. разность $N_{\mathrm{V}}-N_{\mathrm{V} e q}$ меньше, чем $N_{\mathrm{ML}}$, то сначала монослой формируется в быстром режиме за счет вещества в капле до степени заполнения, приближенно равной $\theta_{\max }=\left(N_{\mathrm{V}}-N_{\mathrm{V} e q}\right) / N_{\mathrm{ML}}[6,9]$. Затем наступает „медленный режим“ роста, когда скорость роста монослоя ограничена транспортом атомов V группы в каплю из газовой фазы. Данный режим наблюдается при малых радиусах ННК и/или малом потоке частиц V группы. Эти два сценария реализуются, если граница раздела катализатор-ННК содержит только одну грань кристалла III-V, т. е. фасетирования верхней грани ННК не наблюдается.

Для исследования режимов роста монослоя воспользуемся моделью автокаталитического ПЖК-роста ННК III-V, построенной в работе [8]. Моделирование роста монослоя проводится в два этапа. На первом этапе в рамках классической теории нуклеации моделируется процесс зарождения островка на поверхности заполненного монослоя. Время роста пересыщения оценивается с учетом уравнения материального баланса для частиц $\mathrm{V}$ группы в капле. На втором этапе моделируется латеральный рост монослоя и совместно решаются уравнение материального баланса и уравнение для скорости роста монослоя.

На рис. 1 представлены результаты моделирования роста НHK GaAs, имеющих структуру сфалерита, в направлении [111] при различных плотностях суммарного потока частиц $\mathrm{V}$ группы в каплю $j_{\mathrm{V}}^{t o t}$, радиусе ННК $R_{0}$ и температуре роста $T$. В расчете было использовано значение межфазной энергии границы островка, 


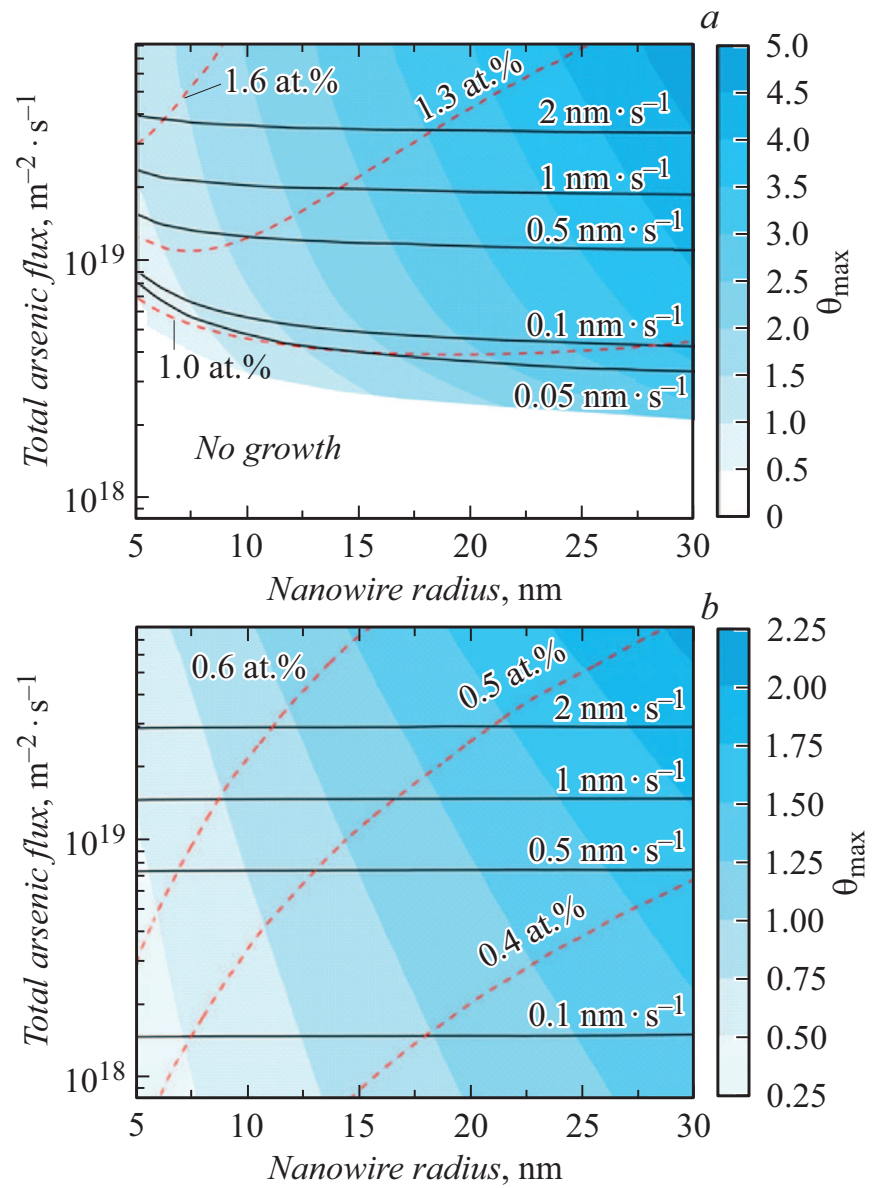

Рис. 1. Зависимость $\theta_{\max }$, скорости роста ННК GaAs (сплошные линии) и мольной доли мышьяка в капле (штриховые линии) от величины потока мышьяка и радиуса ННК при температуре роста $600(a)$ и $500^{\circ} \mathrm{C}(b)$.

полученное в работе [8]: $\gamma=0.394 \mathrm{~J} \cdot \mathrm{m}^{-2}$. Контактный угол капли и угол падения потока мышьяка равны $\beta=125^{\circ}$ и $\alpha_{\mathrm{V}}=35^{\circ}$ [11]. Температурная зависимость коэффициента диффузии мышьяка в галлии взята из работы [12]: $D_{\mathrm{V}}=D_{\mathrm{V} 0} \exp \left(-E_{\mathrm{V}} / k_{\mathrm{B}} T\right)$, где $E_{\mathrm{V}}=0.7 \mathrm{eV}$, $D_{\mathrm{V} 0}=1.59 \cdot 10^{-5} \mathrm{~m}^{2} \cdot \mathrm{s}^{-1}, k_{\mathrm{B}}-$ постоянная Больцмана. Подгоночный коэффициент для скорости роста островка равен $r_{0}=h / \pi[8]$ ( $h$ - высота монослоя). В области, где величина $\theta_{\max }=\left(N_{\mathrm{V}}-N_{\mathrm{V} e q}\right) / N_{\mathrm{ML}}$ меньше единицы, реализуется медленный режим роста (в отсутствие фасетирования верхней грани ННК). Отметим, что пониженное содержание атомов V группы в капле может быть причиной фасетирования из-за растворения верхней грани ННК [4]. С уменьшением температуры область с $\theta_{\max }<1$ увеличивается, так как уменьшается растворимость частиц $\mathrm{V}$ группы в галлии. С увеличением потока $j_{\mathrm{V}}^{t o t}$ увеличиваются концентрация частиц V группы в капле и, следовательно, величина $\theta_{\max }$. Проводя размерный анализ, можно показать $[6,9]$, что величина $\theta_{\max }$ зависит от радиуса ННК как $\theta_{\max } \propto\left(C_{\mathrm{V}}\left(R_{0}\right)-C_{\mathrm{V} e q}(T)\right) R_{0}$, где $C_{\mathrm{V}}$ и $C_{\mathrm{V} e q}-$ максимальная и равновесная мольные доли частиц $\mathrm{V}$ группы в капле. Поскольку $C_{\mathrm{V}}$ слабо зависит от $R_{0}$ (если радиус $R_{0}$ не очень мал), для простых оценок можно аппроксимировать зависимость $\theta_{\max }$ от $R_{0}$ линейной функцией. Этот факт хорошо согласуется с результатами моделирования. При малых $R_{0}$ из-за размерного эффекта увеличивается поток десорбции из капли, и в результате наблюдается резкое уменьшение концентрации атомов V группы $[6,11]$. В области больших значений $j_{\mathrm{V}}^{\text {tot }}$ и $R_{0}$ мольная доля $C_{\mathrm{V}}$ уменьшается с ростом $R_{0}[8,11]$. В этом случае скорость роста ННК слабо зависит от $R_{0}$, так как мало влияние потока десорбции. Это в свою очередь указывает на то, что характерное время нуклеации $\tau_{N}$ также слабо зависит от $R_{0}$. С учетом формулы $\tau_{N}=1 / \pi R_{0}^{2} I$, где $I-$ интенсивность нуклеации, находим, что величина $I$ и, следовательно, $C_{\mathrm{V}}$ должны уменьшаться с ростом $R_{0}$. При высоких температурах роста и/или низких потоках $j_{\mathrm{V}}^{\text {tot }}$ существует область, в которой роста ННК фактически не наблюдается. На рис. 1, $a$ белым цветом выделена область со скоростью роста ННК менее $0.005 \mathrm{~nm} \cdot \mathrm{s}^{-1}$. В данной области суммарный поток частиц V группы из газовой фазы в каплю приближенно равен потоку десорбции частиц $\mathrm{V}$ группы из капли: $j_{\mathrm{V}}^{\text {tot }} S_{\mathrm{V}}=k_{\mathrm{V}} C_{\mathrm{V}}^{2} S_{d}$, где $S_{\mathrm{V}}$ - эффективное сечение капли, которое пересекает поток частиц V группы; $S_{d}$ - площадь поверхности капли; $k_{\mathrm{V}}$ - коэффициент десорбции, зависящий от $C_{\mathrm{V}}$, $R_{0}$ и $T$. Равенство потоков определяет вид изолиний $C_{\mathrm{V}}=$ const в данной области.

Плотность суммарного потока атомов V группы в каплю $j_{\mathrm{V}}^{\text {tot }}$ выражается через эквивалентное давление потока (BEP) частиц V группы согласно формуле $[8,11]$ : $j_{\mathrm{V}}^{\text {tot }}=\left(1+\varepsilon_{\mathrm{V}}\right) \eta p_{\mathrm{V}}$, где $p_{\mathrm{V}}-\mathrm{BEP}$ потока частиц $\mathrm{V}$ группы; $\varepsilon_{\mathrm{V}}$ - коэффициент переиспарения частиц $\mathrm{V}$ группы от подложки и соседних ННК; $\eta-$ коэффициент пересчета, определенный по формуле $\eta=j_{\mathrm{V}}^{d i r} / p_{\mathrm{V}}[13]$; $j_{\mathrm{V}}^{d i r}-$ плотность прямого потока атомов V группы вблизи подложки. В рамках моделей $[8,11]$ путем сравнения экспериментальной [13] и теоретической скорости роста ННК было получено значение $\varepsilon_{\mathrm{V}}$, близкое к 3. В работах $[8,11]$ использовалось значение $\eta=2.3 \cdot 10^{24} \mathrm{~m}^{-2} \cdot \mathrm{s}^{-1} \cdot \mathrm{Torr}^{-1}$ для молекул $\mathrm{As}_{4}$. Однако величина $\eta$ зависит от конфигурации МПЭ-установки и калибровки датчика давления [13]. Так, например, коэффициент пересчета для потока $\mathrm{As}_{4}$ в эксперименте [14] равен $1.28 \cdot 10^{24} \mathrm{~m}^{-2} \cdot \mathrm{s}^{-1} \cdot \mathrm{Torr}^{-1}$. Абсолютное значение потока частиц $\mathrm{V}$ группы определялось путем нахождения величины данного потока, при которой происходит смена режима роста планарных слоев GaAs [14] и GaP [15] с Gа-лимитированного режима на V-лимитированный режим. Из сравнения экспериментальной скорости роста ННК [14] с результатами моделирования находим (при $\gamma=0.394 \mathrm{~J} \cdot \mathrm{m}^{-2}$ ), что коэффициент переиспарения частиц мышьяка приближенно равен 7. В таблице приведены условия роста НHK GaAs и GaP.

На рис. 2 представлены результаты моделирования роста НHК GaP со структурой сфалерита в направ- 
Значение коэффициента переиспарения $\varepsilon_{\mathrm{V}}$ в зависимости от условий роста нитевидных нанокристаллов $\mathrm{GaAs}$ и GaP [14,15] $(t-$ время роста, $V$ - скорость аксиального роста)

\begin{tabular}{|c|c|c|c|c|c|c|c|c|}
\hline$T,{ }^{\circ} \mathrm{C}$ & $t, \min$ & $V, \mathrm{~nm} \cdot \mathrm{s}^{-1}$ & $R_{0}, \mathrm{~nm}$ & $\beta,{ }^{\circ}$ & $\mathrm{BEP}_{\mathrm{Ga}}$, Torr & $\mathrm{BEP}_{\mathrm{v}}$, Torr & $\alpha_{\mathrm{V}},{ }^{\circ}$ & $\varepsilon_{\mathrm{V}}$ \\
\hline \multicolumn{9}{|c|}{ GaAs } \\
\hline 570 & 10 & 0.89 & 51 & $\sim 120$ & $1.25 \cdot 10^{-7}$ & $1.5 \cdot 10^{-6}$ & 30 & 7.0 \\
\hline 600 & 30 & 1.69 & 41 & $\sim 120$ & $1.25 \cdot 10^{-7}$ & $3.0 \cdot 10^{-6}$ & 30 & 7.2 \\
\hline \multicolumn{9}{|c|}{$\mathrm{GaP}$} \\
\hline 610 & 60 & 0.80 & 104 & $\sim 122$ & $1.6 \cdot 10^{-7}$ & $1.92 \cdot 10^{-6}$ & 30 & 5.8 \\
\hline 610 & 60 & 1.98 & 81 & $\sim 122$ & $1.6 \cdot 10^{-7}$ & $2.88 \cdot 10^{-6}$ & 30 & 7.2 \\
\hline 630 & 60 & 2.54 & 42 & $\sim 122$ & $1.6 \cdot 10^{-7}$ & $3.84 \cdot 10^{-6}$ & 30 & 11.1 \\
\hline
\end{tabular}

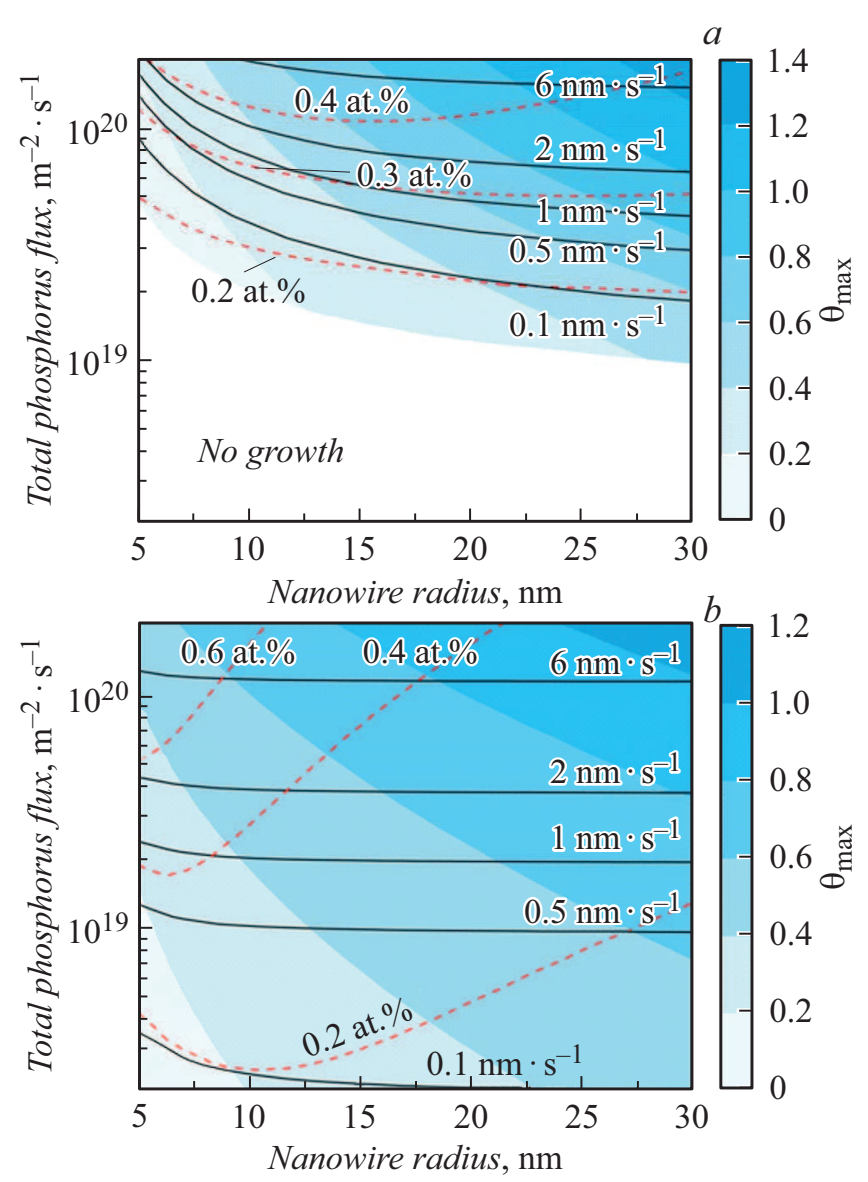

Рис. 2. Зависимость $\theta_{\max }$, скорости роста ННК GaP (сплошные линии) и мольной доли фосфора в капле (штриховые линии) от величины потока фосфора и радиуса ННК при температуре роста $600(a)$ и $500^{\circ} \mathrm{C}(b)$.

лении [111]. При построении графиков использовано значение межфазной энергии для островков $\mathrm{GaP}$ $\gamma=0.47 \mathrm{~J} \cdot \mathrm{m}^{-2}$. Данное значение получено на основе оценки отношения поверхностных энергий кристаллов $\mathrm{GaP}$ и $\mathrm{GaAs}$ (около $1.2[16]$ ) и межфазной энергии для островков $\mathrm{GaAs}$ [8]. Моделирование проводилось при $\beta=120^{\circ}, \alpha_{\mathrm{V}}=30^{\circ}$ и коэффициенте диффузии фосфора, определенном в работе [12]: $E_{\mathrm{V}}=1.69 \mathrm{eV}$,
$D_{\mathrm{V} 0}=4.36 \cdot 10^{-2} \mathrm{~m}^{2} \cdot \mathrm{s}^{-1}$. Отметим, что в настоящей работе предполагается, что диффундируют атомы фосфора. Однако вопрос о сорте частиц фосфора, диффундирующих в жидком галлии, требует дальнейшего изучения. Вид зависимостей $\theta_{\max }, C_{\mathrm{V}}$ и скорости роста ННК от условий роста для систем GaAs и $\mathrm{GaP}$ качественно совпадает. В системе $\mathrm{GaP}$ граница области, в которой рост ННК не наблюдается, сдвигается в область больших потоков, так как десорбционный поток фосфора $\left(\mathrm{P}_{2}\right)$ выше десорбционного потока мышьяка $\left(\mathrm{As}_{2}\right)$. Также по причине большего потока десорбции скорость роста НHК GaP ниже, чем в случае НHК GaAs (при одинаковом значении $j_{\mathrm{V}}^{\text {tot }}$ ). Вместе с тем мольная доля фосфора в галлиевом катализаторе в несколько раз меньше, чем мольная доля мышьяка при росте HНK GaAs. Поэтому область существования медленного режима роста монослоя $\left(\theta_{\max }<1\right)$ существенно больше.

Для получения значений коэффициента переиспарения фосфора было проведено сравнение экспериментальных данных по скорости роста $\mathrm{Ga}$ каталитических НHК GaP [15] с результатами моделирования (см. таблицу). В работе [15] рост ННК производился методом МПЭ на подложках $\mathrm{Si}(111)$ при температуре $610-630^{\circ} \mathrm{C}$. Значение коэффициента пересчета ВЕР потока молекул $\mathrm{P}_{2}$ составило $\eta=2.7 \cdot 10^{24} \mathrm{~m}^{-2} \cdot \mathrm{s}^{-1} \cdot$ Torr $^{-1}$. Рассчитанное значение коэффициента переиспарения фосфора при росте ННК $\mathrm{GaP}$ лежит в интервале значений $\varepsilon_{\mathrm{V}}=6-11$. Большой разброс в значениях $\varepsilon_{\mathrm{V}}$ связан с разбросом в значениях поверхностной плотности ННК.

Таким образом, в работе исследованы режимы роста монослоя Ga-каталитических HНK GaAs и GaP. Полученные зависимости максимальной степени заполнения монослоя и скорости роста ННК могут быть использованы для оптимизации ПЖК-роста ННК в in situ ПЭМ-исследованиях.

\section{Финансирование работы}

Исследование выполнено в рамках проекта Российского научного фонда № 19-72-30004. 


\section{Конфликт интересов}

Авторы заявляют, что у них нет конфликта интересов.

\section{Список литературы}

[1] C.-Y. Wen, J. Tersoff, K. Hillerich, M.C. Reuter, J.H. Park, S. Kodambaka, E.A. Stach, F.M. Ross, Phys. Rev. Lett., 107 (2), 025503 (2011). DOI: 10.1103/PhysRevLett.107.025503

[2] D. Jacobsson, F. Panciera, J. Tersoff, M.C. Reuter, S. Lehmann, S. Hofmann, K.A. Dick, F.M. Ross, Nature, 531 (7594), 317 (2016). DOI: 10.1038/nature17148

[3] J.-C. Harmand, G. Patriarche, F. Glas, F. Panciera, I. Florea, J.-L. Maurice, L. Travers, Y. Ollivier, Phys. Rev. Lett., 121 (16), 166101 (2018). DOI: 10.1103/PhysRevLett.121.166101

[4] F. Panciera, Z. Baraissov, G. Patriarche, V.G. Dubrovskii, F. Glas, L. Travers, U. Mirsaidov, J.-C. Harmand, Nano Lett., 20 (3), 1669 (2020). DOI: 10.1021/acs.nanolett.9b04808

[5] C.B. Maliakkal, E.K. Mårtensson, M.U. Tornberg, D. Jacobsson, A.R. Persson, J. Johansson, L.R. Wallenberg, K.A. Dick, ACS Nano, 14 (4), 3868 (2020). DOI: 10.1021/acsnano.9b09816

[6] F. Glas, V.G. Dubrovskii, Phys. Rev. Mater., 4 (8), 083401 (2020). DOI: 10.1103/PhysRevMaterials.4.083401

[7] В.Г. Дубровский, А.С. Соколовский, И.В. Штром, Письма в ЖТФ, $46 \quad$ (18), 3 (2020). DOI: $10.21883 /$ pjtf.2020.18.49991.18401 [V.G. Dubrovskii, A.S. Sokolovskii, I.V. Shtrom, Tech. Phys. Lett., 46 (9), 889 (2020). DOI: 10.1134/S1063785020090187].

[8] A.A. Koryakin, S.A. Kukushkin, Phys. Status Solidi B, 258 (6), 2000604 (2021). DOI: 10.1002/pssb.202000604

[9] В.Г. Дубровский, Письма в ЖТФ, 46 (8), 3 (2020). DOI: $10.21883 /$ pjtf.2020.08.49298.18204 [V.G. Dubrovskii, Tech. Phys. Lett., 46 (4), 357 (2020). DOI: $10.1134 / \mathrm{S} 1063785020040203]$.

[10] V.G. Dubrovskii, Cryst. Growth Des., 17 (5), 2589 (2017). DOI: 10.1021/acs.cgd.7b00124

[11] F. Glas, M.R. Ramdani, G. Patriarche, J.-C. Harmand, Phys. Rev. B, 88 (19), 195304 (2013). DOI: $10.1103 /$ PhysRevB.88.195304

[12] V.A. Gorokhov, T.T. Dedegkaev, Y.L. Ilyin, V.A. Moshnikov, A.S. Petrov, Y.M. Sosov, D.A. Yaskov, Cryst. Res. Technol., 19 (11), 1465 (1984). DOI: 10.1002/crat.2170191112

[13] M.R. Ramdani, J.C. Harmand, F. Glas, G. Patriarche, L. Travers, Cryst. Growth Des., 13 (1), 91 (2013). DOI: $10.1021 / \mathrm{cg} 301167 \mathrm{~g}$

[14] A.D. Bolshakov, V.V. Fedorov, N.V. Sibirev, M.V. Fetisova, E.I. Moiseev, N.V. Kryzhanovskaya, O.Y. Koval, E.V. Ubyivovk, A.M. Mozharov, G.E. Cirlin, I.S. Mukhin, Phys. Status Solidi (RRL), 13 (11), 1900350 (2019). DOI: $10.1002 /$ pssr.201900350

[15] V.V. Fedorov, Y. Berdnikov, N.V. Sibirev, A.D. Bolshakov, S.V. Fedina, G.A. Sapunov, L.N. Dvoretckaia, G. Cirlin, D.A. Kirilenko, M. Tchernycheva, I.S. Mukhin, Nanomaterials, 11 (8), 1949 (2021). DOI: 10.3390/nano11081949

[16] S. Mirbt, N. Moll, K. Cho, J.D. Joannopoulos, Phys. Rev. B, 60 (19), 13283 (1999). DOI: 10.1103/PhysRevB.60.13283 